\title{
Assessing manual lifting tasks based on segment angle interpolations
}

\author{
Chien-Chi Chang ${ }^{\mathrm{a}, *}, \mathrm{Xu} \mathrm{Xu^{ \textrm {a } } \text { , Gert S. Faber, }}{ }^{\mathrm{a}, \mathrm{b}}$, Idsart Kingma ${ }^{\mathrm{c}}$ and Jack Dennerlein ${ }^{\mathrm{b}}$ \\ ${ }^{a}$ Liberty Mutual Research Institute for Safety, 71 Frankland Road, Hopkinton, MA 01748, USA \\ ${ }^{\mathrm{b}}$ Department of Environmental Health, Harvard School of Public Health, 665 Huntington Avenue, Boston, MA \\ 02139, USA \\ ${ }^{\mathrm{c}}$ Institute for Fundamental and Clinical Human Movement Sciences, Faculty of Human Movement Sciences, Vrije \\ Universiteit, Van der Boechorststraat 9, 1081 BT Amsterdam, The Netherlands
}

\begin{abstract}
This study investigates the effects of the number of interpolation points on the prediction accuracy of segment angle trajectory during lifting. Ten participants performed various lifting tasks while a motion tracking system recorded their movements. Two-point through ten-point equal time-spaced segment angles extracted from major segment trajectory data captured by the motion tracking system were used to re-generate the whole body lifting motion by using polynomial and cubic spline interpolation methods. The root mean square error (RMSE) between the reference (motion tracking system) and the estimated (interpolation method) segment angle trajectories were calculated to quantify the prediction accuracy. The results showed that the cubic spline interpolation will yield a smaller RMSE value than one based on the polynomial interpolation. While increasing the number of interpolation points can reduce the RMSE of the estimated segment angle trajectories, there was a diminishing advantage in continuing to add interpolation points. A sensitivity analysis suggests that if the estimation of the segment angles at each interpolation point deviates considerably from the real value, and cannot be controlled at a low level $\left(<10^{\circ}\right)$, the use of higher number of interpolation points will not improve the estimation accuracy.
\end{abstract}

Keywords: simulation, joint trajectory, posture coding

\section{Introduction}

Multiple-linked segment biomechanical models have often been used in research by ergonomists to quantify and evaluate the movements and joint loadings of manual lifting tasks. To assess the kinematics and kinetics of manual lifting tasks, displacementtime data for the various body segments of interest must be known.

Previous research studies have examined the use of interpolation methods to generate a plausible continuous movement trajectory from separate postures for computer animation and virtual reality applications [1-3]. For estimating the dynamic motion of lifters, studies $[4,5]$ have proposed a computerized video posture coding approach for predicting the detailed body segment movements over the course of lifting tasks from field survey videos. Field survey videos are easy to obtain at the workplace and are actually capable of providing much valuable information, including time and duration of the lifting task configuration. It can also provide key lifting postures by slow motion playback. The main idea of a video posture coding approach was that the subject's segment angle movement could be estimated and regenerated by a polynomial regression based on coderidentified major segment angles of selected key lifting postures extracted from the side-view video clips of the lifting task. With the advent of such simulation models, it is possible to describe and assess lifting tasks when the use of a motion tracking system is limited or unavailable due to practical restrictions. However, in the earlier research studies, the relationship between the minimum required number of interpolation key lifting posture frames and the estimation accuracy of segment angle trajectories was unclear and has not been systematic investigated in detail. Additionally, it has been proven that the polynomial

\footnotetext{
*Corresponding author. E-mail: chien-chi.chang@libertymutual.com.
} 
interpolation algorithm is not convergent as the number of interpolation node increases. Also not clarified was whether the use of different interpolation algorithms, such as cubic spline interpolation method, could provide a better prediction model in comparison to the polynomial interpolation algorithm that was originally used in earlier studies.

In the current study, the effects of the interpolation algorithms and number of interpolation points on the prediction accuracy are further investigated. In addition, a sensitivity analysis was also performed to investigate how the root mean square error (RMSE) changed if the segment angles used for the interpolations randomly deviated from the actual ones (to simulate the error due to imperfect posture estimation of the segment angle).

\section{Method}

\subsection{Participants}

Ten male participants in good health and free of musculoskeletal disorders were recruited to participate in this study. The means (standard deviations) of participants age, height, and weight were 23 (2.5) years, $184(6) \mathrm{cm}$, and $76.1(6.3) \mathrm{kg}$, respectively. The experimental procedure was approved by the appropriate internal review board. The study protocol was explained to each participant prior to the experiment. The experiment took place after a participant reviewed and signed the informed consent form and agreed to participate.

\subsection{Procedure}

Participants were asked to perform a variety of lifting tasks. A box (width $55 \mathrm{~cm}$, depth $35 \mathrm{~cm}$, height $33 \mathrm{~cm}$ ) was used in the experiment for the lifting tasks. Weight was added to the box as necessary to produce different loading conditions. A motion tracking system (OptotrakT ${ }^{\mathrm{M}}$, Northern Digital, Waterloo, Canada) with two arrays of three cameras was used to record the participant's segment movement data. The capture rate was set at 100 samples per second. The LED markers of the motion tracking system were attached to the center of sagittal plane projection of a participant's major segments. The order of lifting conditions was randomized. The testing conditions included two lifting techniques (stoop and squat), three lifting ranges (initial load position
50,250 and $550 \mathrm{~mm}$ from ground), and six load weight levels $(1.9,6.8,11.9,16.9,21.9$ and $26.6 \mathrm{~kg})$.

\subsection{Model and analysis}

A rigid link model with seven body segments (foot, shank, thigh, pelvis, trunk, upper arm and forearm) was adopted in this study. For each participant, the segment movement data collected from the motion tracking system over the course of each lifting task were used to calculate the angular displacement-time trajectories of major segments based on this rigid link model. The results of these calculations were referred to as the "measured" segment angle trajectories. Two-point through ten-point equal time-spaced segment angles extracted from the above measured data were used to re-generate the whole body lifting motion using two interpolation algorithms: polynomial and cubic spline. The output results were referred to as "estimated" segment angle trajectories. The root mean square error (RMSE) between the measured and the estimated segment angle trajectories were calculated to examine the disagreement between the data calculated from the motion tracking system and the data generated by both interpolation methods.

To analyze how the RMSE may change when the segment angles used for the input points of the interpolation algorithm deviated from the measured ones (to mimic the anticipated error when using a video posture coding method to estimate the key segment angles), a sensitivity analysis was performed. A random number with a uniform distribution ranging within $\pm 2.5^{\circ}, \pm 5^{\circ}, \pm 10^{\circ}$, and $\pm 15^{\circ}$ was added to the accurate interpolation points before the interpolation was performed. Then, the RMSE the of estimated segment angle trajectory was calculated for each segment angle.

\section{Results and discussion}

The RMSE of each segment angle decreased for both interpolation algorithms (Figure 1) when the number of interpolation points increased from 2 points to 8 points. For 2-point to 4-point interpolation, the RMSEs were the same for the two interpolation algorithms. This was because cubic spline is a series of 3rd order polynomials which yields the same interpolation when the number of interpolation point is less than 5. For 5-point to 10-point, the RMSE of the cubic spline interpolation was smaller than the 
RMSE of the polynomial interpolation method. When the number of interpolation points exceeded 8 , adding more interpolation points increased the RMSE for the polynomial interpolation algorithm. Overall, if a 4-point polynomial or cubic spline interpolation is applied, the RMSE can be maintained at less than 2 degrees for all segments. To achieve a precise estimation with RMSE values smaller than 0.5 degree for all segment angles, a 7 or 8 points cubic spline interpolation will be required.
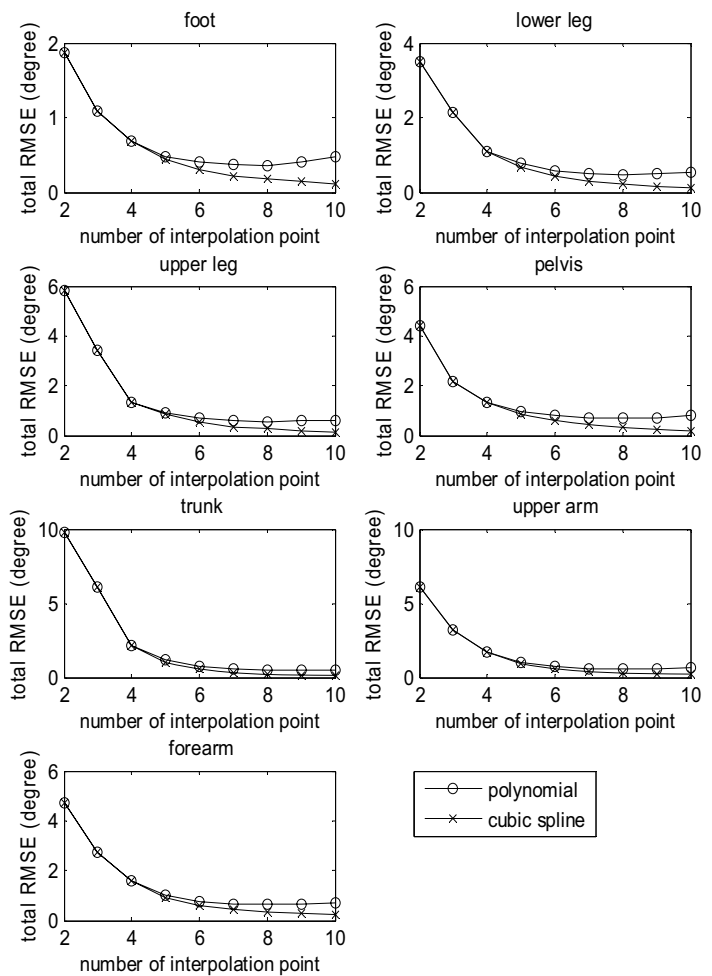

Fig. 1. The root mean square error (RMSE) of each body segment with different interpolation algorithms and numbers of interpolation points

The results indicated that, while increasing the number of interpolation points in the cubic spline approach can reduce the RMSE of the estimated segment angle trajectories, the decrease of the RMSE appears to be non-linear. As the number of points increased, the improvement of adding points on interpolation became smaller. There was a diminishing advantage in continuing to add interpolation points beyond a certain number. The results also revealed that the cubic spline approach had better convergence than the polynomial on the estimation of segment angle trajectories. With fewer than 8 interpolation points, the polynomial approach had similar results. However, its RMSE started to increase when the number of interpolation points was too high because of Runge's phenomenon.

Since cubic spline interpolation always had the same or better estimation on segment angle trajectories than polynomial interpolation, the sensitivity analysis was performed only with the cubic spline interpolation algorithm used. The analysis results (Figure 2) showed that when a random error with uniform distribution ranging within $\pm 5^{\circ}$ was added to the interpolation points, the RMSE did not significantly reduce with more than 5 interpolation points used. For the random error ranges at $\pm 10^{\circ}$ or $\pm 15^{\circ}$, benefits for improving prediction accuracy by increasing the number of interpolation points diminished.
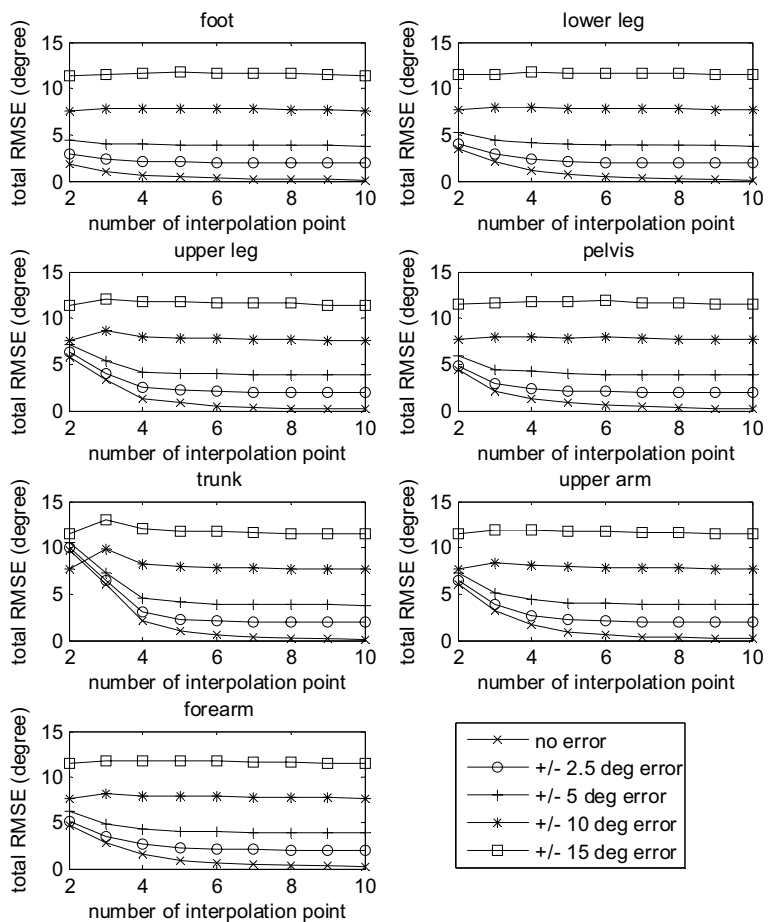

Fig. 2. The root mean square error (RMSE) of each interpolated body segment angle when the sensitivity analysis imposed random errors on the identified interpolation points segment angles (to mimic the anticipated error when using a video posture coding method to estimate the key segment angles used for the input points of the interpolation algorithm) 
The results of the sensitivity analysis also imply that, in order to accurately regenerate the segment angle trajectories when applying these interpolation approaches, the error on the segment angles at each interpolation point should be controlled to a low level. If the interpolation points considerably deviate from the real value (random errors up to 10 degrees or more), increasing the number of interpolation points may not necessarily help to improve estimation accuracy. This should be particularly considered when the body segment angle is derived from posture observation since this method can introduce substantial perception errors [6]. Future research should focus on how to control perception errors of segment angles during posture observation.

\section{Conclusions}

With the use of interpolation algorithms, the segment angle trajectories during lifting tasks can be regenerated with data from only a few key body postures. The results suggest that cubic spline interpolation provides a better estimation of segment angle trajectories compared with polynomial interpolation. To ensure a good trajectory estimate, the error of segment angle inputs due to the use of video posture coding in each interpolation point needs to be controlled at a low level. With further research and improvement of current methods, it is possible to describe or assess lifting tasks when the use of complex motion tracking systems is limited or unavailable due to practical considerations.

\section{References}

[1] Badler, N.I., Bindiganavale, R., Granieri, J.P., Wei, S., Zinmin, Z., 1994, Posture interpolation with collision avoidance. Proceedings of Computer Animation '94, IEEE, 13-20 (May).

[2] Rose, C., Cohen, M.F., Bodenheimer, B., 1998. Verbs and adverbs: multidimensional motion interpolation. IEEE Computer Graphics and Applications. 18, 32-40.

[3] Mori, H., Hoshino, J., 2003, ICA-based interpolation of human motion. Proceedings of IEEE International Symposium on Computational Intelligence in Robotics and Automation, IEEE, Kobe, Japan.

[4] Chang, C.C., McGorry, R.W., Lin, J.H., Xu, X., Hsiang, S., 2010, Prediction accuracy in estimating joint angle trajectories using a video posture coding method for sagittal lifting tasks. Ergonomics, 53, 1039-1047.

[5] Chang, C.C., Hsiang, S.M., Dempsey, P.G., McGorry, R.W., 2003, A computerized video-based biomechanical analysis tool for lifting tasks. International Journal of Industrial Ergonomics, 32 (4), 239-250

[6] deLooze, M.P., Toussaint, H.M., Ensink, J., Mangnus, C., van der Beek, A.J., 1994, The validity of visual observation to assess posture in a laboratory-simulated, manual material handling task. Ergonomics, 37, 1335-1343. 